Communi cat $i$ on: Act $i$ vat $i$ on ener gy of tensi on- $i$ nduced pore for mat $i$ on i $n$ I i pi d menbr anes

\begin{tabular}{|c|c|}
\hline 著者 & Kar al Nohammad Abu Sayem Yamazaki Nasahi to \\
\hline $\begin{array}{l}\text { jour nal or } \\
\text { publ i cat } i \text { on } t i t l e\end{array}$ & The Journal of Chemi cal Physi cs \\
\hline vol une & 143 \\
\hline number & 8 \\
\hline page $r$ ange & 081103 \\
\hline year & 2015-08-31 \\
\hline 出版者 & Amer i can I nst i tute of Physics \\
\hline 権利 & Copyright ( C) 2015 Al P Publ i shi ng LLC \\
\hline URL & ht t p: //hdl . handl e. net /10297/9109 \\
\hline
\end{tabular}




\title{
Communication: Activation energy of tension-induced pore formation in lipid membranes
}

\author{
Mohammad Abu Sayem Karal ${ }^{1}$ and Masahito Yamazaki ${ }^{1,2,3, a)}$ \\ ${ }^{1}$ Integrated Bioscience Section, Graduate School of Science and Technology, Shizuoka University, \\ Shizuoka 422-8529, Japan \\ ${ }^{2}$ Nanomaterials Research Division, Research Institute of Electronics, Shizuoka University, \\ Shizuoka 422-8529, Japan \\ ${ }^{3}$ Department of Physics, Faculty of Science, Shizuoka University, Shizuoka 422-8529, Japan
}

(Received 16 July 2015; accepted 24 August 2015; published online 31 August 2015)

\begin{abstract}
Tension plays a vital role in pore formation in biomembranes, but the mechanism of pore formation remains unclear. We investigated the temperature dependence of the rate constant of constant tension $(\sigma)$-induced pore formation in giant unilamellar vesicles of lipid membranes using an experimental method we developed. By analyzing this result, we determined the activation energy $\left(U_{\mathrm{a}}\right)$ of tensioninduced pore formation as a function of tension. A constant $\left(U_{0}\right)$ that does not depend on tension was found to contribute significantly to $U_{\mathrm{a}}$. Analysis of the activation energy clearly indicated that the dependence of $U_{\mathrm{a}}$ on $\sigma$ in the classical theory is correct, but that the classical theory of pore formation is not entirely correct due to the presence of $U_{0}$. We can reasonably consider that $U_{0}$ is a nucleation free energy to form a hydrophilic pre-pore from a hydrophobic pre-pore or a region with lower lateral lipid density. After obtaining $U_{0}$, the evolution of a pre-pore follows a classical theory. Our data provide valuable information that help explain the mechanism of tension-induced pore formation in biomembranes and lipid membranes. @ 2015 AIP Publishing LLC. [http://dx.doi.org/10.1063/1.4930108]
\end{abstract}

\section{INTRODUCTION}

External force applied to cells or vesicles (liposomes) of lipid membranes induces lateral tension in plasma membranes or lipid membranes. Positive tension induces stretching of the membranes, and when it reaches above critical values, pore formation occurs in the membranes, causing cell death or rupture of vesicles. It is therefore important to elucidate the mechanism of pore formation. Giant unilamellar vesicles (GUVs) composed of lipid membranes have been used to elucidate the kinetics and mechanism of tension-induced pore formation. ${ }^{1-6}$

The classical theory of tension-induced pore formation ${ }^{7,8}$ was based on the theory of how soap films rupture. ${ }^{9}$ According to the classical theory, once a pre-pore with radius $r$ is formed in the membrane, the total free energy of the system changes by an additional free energy component (called the free energy of a pre-pore $U(r, \sigma))$ consisting of two terms: one term $\left(-\pi r^{2} \sigma\right)$ associated with lateral tension $(\sigma)$, favoring expansion of the pre-pore, and the other term $(2 \pi r \Gamma)$ associated with the line tension $(\Gamma)$ of the pre-pore edge, favoring pre-pore closure. The free energy of a pre-pore, $U(r, \sigma)$, can therefore be expressed as follows:

$$
U(r, \sigma)=2 \pi r \Gamma-\pi r^{2} \sigma,
$$

where $U(r, \sigma)$ has a maximum of $U_{\mathrm{a}}=U\left(r_{\mathrm{a}}\right)=\pi \Gamma^{2} / \sigma$ at $r$ $=r_{\mathrm{a}}(=\Gamma / \sigma)$. Thermal fluctuation in the lateral density of a lipid membrane induces a pre-pore. ${ }^{5}$ If the radius of a pre-pore

\footnotetext{
a) Author to whom correspondence should be addressed. Electronic mail: yamazaki.masahito@shizuoka.ac.jp. Telephone/fax: 81-54-238-4741.
}

is less than the critical radius, $r_{\mathrm{a}}(=\Gamma / \sigma)$, it closes quickly. However, if the radius expands and reaches $r_{\mathrm{a}}$, the pre-pore transforms into a pore. This classical theory has been used to explain several phenomena, such as the rate of closure and opening of pores ${ }^{1,2}$ and the tension dependence of the rate constant of tension-induced pore formation. ${ }^{5,6}$

Evans and colleagues found that at a fast loading rate (i.e., a rapid rate of increase in tension), the classical theory could not explain their results regarding the tension-induced rupture of a GUV. Based on their results, they proposed that the rupture of a GUV (i.e., pore formation in a GUV) can be modeled as a sequence of two thermally activated transitions (i.e., they proposed the existence of a metastable intermediate state) ${ }^{3,4}$ However, no direct experimental evidence of a defect in the classical theory or the existence of an intermediate state has been published to date.

In this study, we investigated the temperature dependence of the rate constant of constant tension-induced pore formation in GUVs using a method we developed ${ }^{5}$ in order to validate the classical theory and the Evans model experimentally. Based on our results, we determined the activation energy of tension-induced pore formation as a function of tension. The dependence of the activation energy on tension indicated that a constant which does not depend on tension contributes significantly to the activation energy.

\section{MATERIALS AND METHODS}

Dioleoylphosphatidylcholine (DOPC) was purchased from Avanti Polar Lipids Inc. (Alabaster, AL). Bovine serum 
albumin (BSA) was purchased from Wako Pure Chemical Industry Ltd. (Osaka, Japan). DOPC-GUVs were prepared by natural swelling in water (MilliQ) containing $0.1 \mathrm{M}$ sucrose. ${ }^{5}$ DOPC-GUV suspensions ( $300 \mu \mathrm{L})(0.1 \mathrm{M}$ sucrose in water as the internal solution; $0.1 \mathrm{M}$ glucose in water as the external solution) were transferred into a hand-made microchamber. ${ }^{10}$ GUVs were observed using an inverted fluorescence phasecontrast and differential interference contrast (DIC) microscope (IX-71, Olympus, Tokyo, Japan) at various temperatures controlled via a stage thermocontrol system (ThermoPlate CBU, TP-CH110R-C, Tokai Hit, Shizuoka, Japan). Phasecontrast and DIC images of GUVs were recorded using a charge-coupled device (CCD) camera (CS230B, Olympus).

The rate constant of constant tension-induced pore formation in a GUV was determined using the method of Levadny et al. ${ }^{5}$ To apply tension, $\sigma$, to the lipid membranes of single GUVs, we used the micropipette aspiration method. ${ }^{11} \sigma$ can be described as a function of the difference in pressure between the outside and the inside of a micropipette, $\Delta P$, as follows: ${ }^{11}$

$$
\sigma=\frac{\Delta P d}{4(1-d / D)},
$$

where $d$ represents the internal diameter of the micropipette and $D$ represents the diameter of the spherical portion of the GUV exterior to the micropipette. Micropipettes were coated with $0.5 \%(\mathrm{w} / \mathrm{v}) \mathrm{BSA}$ in $0.1 \mathrm{M}$ glucose, and glass surfaces in chambers were coated with $0.1 \%(\mathrm{w} / \mathrm{v}) \mathrm{BSA}$ in $0.1 \mathrm{M}$ glucose.

\section{RESULTS}

We measured the temperature dependence of the rate constant of tension-induced pore formation in GUVs of DOPC (i.e., DOPC-GUVs). First, we used a micropipette to apply a constant tension of $8.0 \mathrm{mN} / \mathrm{m}$ to a DOPC-GUV at $12{ }^{\circ} \mathrm{C}$ in $0.1 \mathrm{M}$ glucose solution for a specific amount of time until rupture of the GUV was observed. For this experiment, we initially held a single GUV at the tip of a micropipette for 2 min using only slight aspiration pressure, with a tension on the bilayer of $\sim 0.5 \mathrm{mN} / \mathrm{m}$. We then rapidly $(\sim 10 \mathrm{~s})$ increased the pressure difference, $\Delta P$, for aspiration to obtain a specific level of tension, $\sigma$ (here, $\sigma=8.0 \mathrm{mN} / \mathrm{m}$ ), and maintained this tension. After $74 \mathrm{~s}$ passed, the GUV was suddenly aspirated into the micropipette. The time of pore formation was defined as the point at which the GUV was completely aspirated and was recorded with a resolution of less than $1 \mathrm{~s}$. The process leading to aspiration of the GUV into the micropipette can be explained as follows: ${ }^{5}$ First, a pore was formed in the GUV membrane, leading to rupture of the GUV; the GUV was then completely aspirated into the micropipette due to the pressure difference between the inside and the outside of the micropipette. When we repeated the same experiment with many single GUVs $(n=20)$, we found that pore formation occurred stochastically after the elapse of different amounts of time. The change over time in the fraction of intact GUVs without rupture among all of the GUVs examined, $P_{\text {intact }}(t)$, fit a single exponential decay function, as follows (Fig. 1(a)):

$$
P_{\text {intact }}(t)=\exp \left(-k_{\mathrm{p}} t\right)
$$

where $k_{\mathrm{p}}$ represents the rate constant of tension-induced rupture of a GUV, which is the same as the rate constant of tension-induced pore formation, and $t$ represents the duration of the continuous tension applied to the GUV (i.e., continuous tension beginning at $t=0$ ). From this fitting, we obtained the value of $k_{\mathrm{p}}: 7.3 \times 10^{-3} \mathrm{~s}^{-1}$.

Next, we performed the same experiment at $32{ }^{\circ} \mathrm{C}(n$ $=20)$. At $32^{\circ} \mathrm{C}$, the decrease in $P_{\text {intact }}(t)$ occurred more rapidly than at $12{ }^{\circ} \mathrm{C}$ (Fig. 1(a)), and the value for $k_{\mathrm{p}}$ obtained from curve fitting was $2.4 \times 10^{-2} \mathrm{~s}^{-1}$. To obtain the activation energy of $k_{\mathrm{p}}$, we plotted $\ln k_{\mathrm{p}}$ versus $1 / T$ (Fig. 1(b)). The activation energy, $U_{\mathrm{a}}$, was obtained from the slope of the resulting linear fit: $U_{\mathrm{a}}=73.7 \pm 1.9 \mathrm{pN} \mathrm{nm}\left(=17.9 \pm 0.5 k_{\mathrm{B}} T\right.$, where $k_{\mathrm{B}}$ represents the Boltzmann constant and the value of $T$ is $298 \mathrm{~K}$ ).

We also measured the temperature dependence of $k_{\mathrm{p}}$ using tensions of 7.0 and $7.5 \mathrm{mN} / \mathrm{m}$. Figure 2(a) shows plots of $\ln k_{\mathrm{p}}$ versus $1 / T$. These plots are linear, and values for $U_{\mathrm{a}}$ were determined from the corresponding slopes: $U_{\mathrm{a}}=81.5$ $\pm 1.9 \mathrm{pN} \mathrm{nm}\left(=19.8 \pm 0.5 k_{\mathrm{B}} T\right)$ for $\sigma=7.0 \mathrm{mN} / \mathrm{m}$ and $U_{\mathrm{a}}$ $=77.1 \pm 1.4 \mathrm{pN} \mathrm{nm}\left(=18.8 \pm 0.4 k_{\mathrm{B}} T\right)$ for $\sigma=7.5 \mathrm{mN} / \mathrm{m}$. The curve for $U_{\mathrm{a}}$ versus $1 / \sigma$ produced a linear line expressed by $U_{\mathrm{a}}=U_{0}+B / \sigma$, where $U_{0}$ and $B$ are constants that do not depend on tension (Fig. 2(b)). From the best fitting of the data, we obtained the value for $U_{0}: 19 \pm 3 \mathrm{pN} \mathrm{nm}\left(=4.7 \pm 0.6 k_{\mathrm{B}} T\right)$. Therefore, the $U_{0}$ fraction in the total activation energy, $U_{\mathrm{a}}$, is large (e.g., 0.26 for $\sigma=8.0 \mathrm{mN} / \mathrm{m}$ ).

\section{DISCUSSIONS}

In this report, we measured the temperature dependence of tension-induced pore formation in DOPC-GUVs, and from
(A)

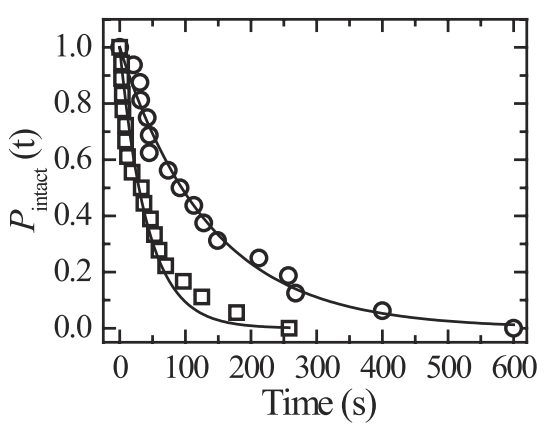

(B)

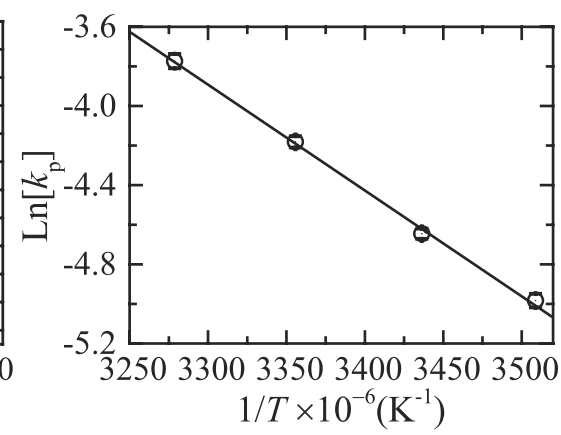

FIG. 1. The effect of temperature on tension-induced pore formation in a single GUV. (a) Time-course of $P_{\text {intact }}(t)$ in the presence of $8.0 \mathrm{mN} / \mathrm{m}$ of tension at $12{ }^{\circ} \mathrm{C}(\mathrm{O})$ and $32{ }^{\circ} \mathrm{C}(\square)$. Solid line represents the best-fit curve for Eq. (3). (b) Relationship between $k_{\mathrm{p}}$ and $1 / T$. Average values and standard error of $k_{\mathrm{p}}$ for each temperature were determined in three independent experiments using 20 GUVs in each experiment. Error bars show standard error. Solid line shows the best-fit linear line. 
(A) (B)
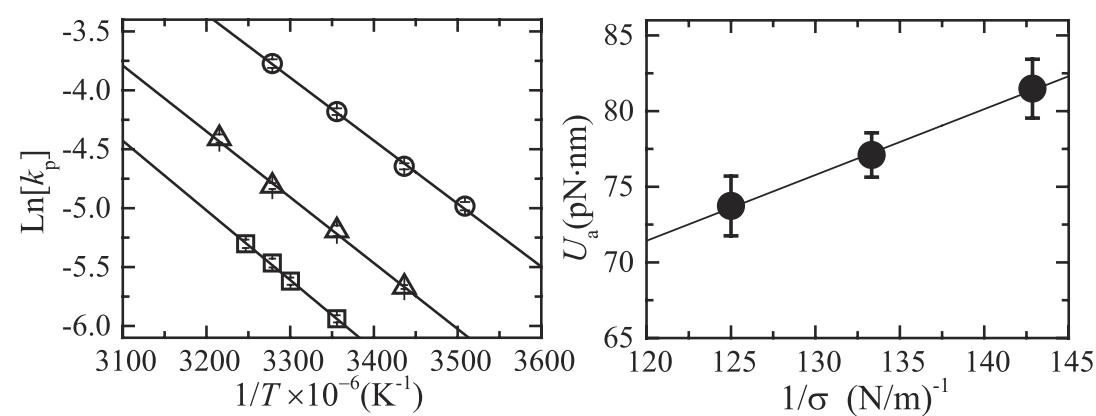

FIG. 2. Tension dependence of the activation energy of tension-induced pore formation. (a) Relationship between $k_{\mathrm{p}}$ and $1 / T$ for $(\square) 7.0 \mathrm{mN} / \mathrm{m},(\Delta)$ $7.5 \mathrm{mN} / \mathrm{m}$, and (O) $8.0 \mathrm{mN} / \mathrm{m}$ of applied tension. Average values and standard error of $k_{\mathrm{p}}$ for each temperature were determined in three independent experiments, each using 20 GUVs. Error bars show standard error. Solid lines show best-fit linear lines. (b) Relationship between $U_{\mathrm{a}}$ and $1 / \sigma$. Error bars show standard error. Solid line shows the best fit in accordance with Eq. (4). these data, we determined the activation energy of pore formation. To the best of our knowledge, this is the first report determining the activation energy of tension-induced pore formation in biomembranes or lipid membranes. We also obtained the dependence of $U_{\mathrm{a}}$ on $\sigma$, and these data indicated that a linear relationship exists between $U_{\mathrm{a}}$ and $1 / \sigma$. The physical implications of these results are discussed below.

As described at the beginning of this report, according to the classical theory, $U_{\mathrm{a}}=\pi \Gamma^{2} / \sigma$. However, the data shown in Figure 2(b) fit a linear line expressed by the equation $U_{\mathrm{a}}$ $=U_{0}+B / \sigma$, where $U_{0}$ and $B$ are constants that do not depend on tension, although this equation holds for the limited range of small values of the tension $(7.0-8.0 \mathrm{mN} / \mathrm{m})$. These data thus clearly indicate that the dependence of $U_{\mathrm{a}}$ on $\sigma$ in the classical theory of pore formation is correct, but that the classical theory of pore formation is not entirely correct due to the presence of the constant term which does not depend on $\sigma$. Based on an examination of the result shown in Figure 2(b) in reference to the classical theory, we propose the following reasonable revision to the theory:

$$
U_{\mathrm{a}}=U_{0}+\pi \Gamma^{2} / \sigma
$$

From the best-fit value for $B$ in the plot shown in Figure 2(b), we obtained the value of $\Gamma: 11.6 \pm 0.2 \mathrm{pN}$. This

(A)

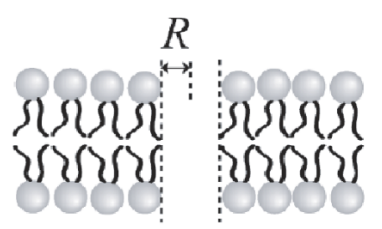

(2)

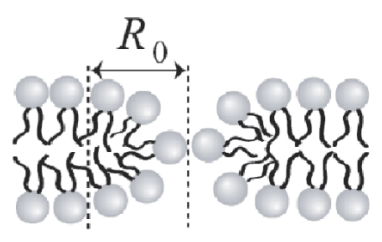

(3)
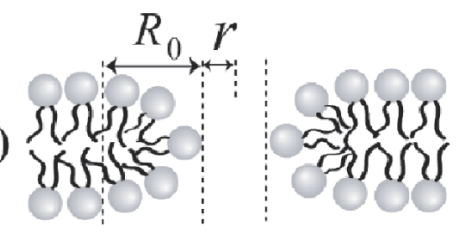

FIG. 3. Illustration of two types of prepores and the free energy profile of a pre-pore as a function of pre-pore radius, $R$. (a-1) A hydrophobic pre-pore with radius of $R$. (a-2) State I between a hydrophobic and a hydrophilic pre-pore at $R=R_{0}$. (a-3) A hydrophilic pre-pore with radius of $r$. (b) Model $\mathrm{A}$ has an energy barrier between the intact and intermediate states. (c) Model B has no energy barrier between the two states.
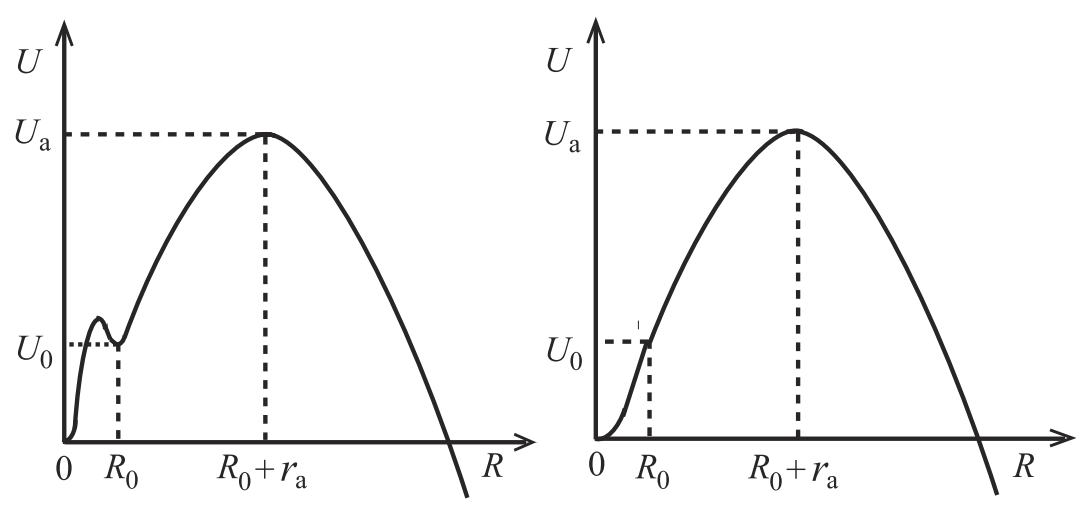
value is almost the same as that $(10.5 \mathrm{pN})$ obtained in an analysis $^{5}$ of the tension dependence of $k_{\mathrm{p}}$ and that obtained by dynamic tension spectroscopy $(11.5 \mathrm{pN}) .{ }^{3}$ Generally, the rate constant for any reaction can be described using its activation energy, which can be converted using Eq. (4) into the following general equation:

$$
\begin{aligned}
k_{\mathrm{p}} & =A \exp \left(-U_{\mathrm{a}} / k_{\mathrm{B}} T\right) \\
& =A \exp \left(-U_{0} / k_{\mathrm{B}} T\right) \exp \left(-\pi \Gamma^{2} / k_{\mathrm{B}} T \sigma\right) .
\end{aligned}
$$

Several reaction schemes may be explained by Eqs. (4) and (5). Here, we consider the evolution of a pre-pore into a pore. Two types of pre-pores have been described: one is a hydrophobic pre-pore in which the wall is composed of the hydrocarbon chains of membrane lipids (Fig. 3(a-1)), and the other is a hydrophilic pre-pore in which the wall is composed of the hydrophilic segments of membrane lipids (Fig. 3(a-3)). ${ }^{12-15}$ When the radius is small, the pre-pore is hydrophobic, but as the radius expands and reaches a critical value, the prepore becomes hydrophilic. ${ }^{12-15}$ Atomistic molecular dynamics simulation results ${ }^{13,14}$ indicate the structure of a hydrophilic pre-pore shown in Fig. 3(b). However, they do not indicate the structure of a hydrophobic pre-pore shown in Fig. 3(a) but rather a region with lower lateral lipid density than the average. We can reasonably consider that $U_{0}$ is the free energy of a state I between a hydrophobic and a hydrophilic pre-pore at $R=R_{0}$ (Fig. 3(a-2)). Two models have been proposed to describe the conversion of hydrophobic pre-pores into hydrophilic prepores. In one model (model A), an energy barrier exists between the intact state $\mathrm{O}$ (at $R=0$ ) and state $\mathrm{I}$ (at $R=R_{0}$ ) (Fig. 3(b)), ${ }^{3,4}$ whereas in the second model (model B), no energy barrier exists between these two states (Fig. 3(c)). ${ }^{13,14}$ In both cases, the free energy profile of the hydrophilic prepore as a function of its radius $r$ can be described by the following equation (Fig. 4):

$$
U(r, \sigma)=U_{0}+2 \pi \Gamma r-\pi \sigma r^{2}
$$

where the radius of the hydrophilic pre-pore in state $\mathrm{I}, r$, is defined as zero. Therefore, $U_{0}$ can be considered a nucleation free energy to form a hydrophilic pre-pore from a hydrophobic pre-pore or a region with lower lateral lipid density. After obtaining $U_{0}$, the evolution of a pre-pore follows a classical theory. The activation energy of the transition from state I at $r$ $=0$ to the pore state $\mathrm{P}$ is $U\left(r_{\mathrm{a}}\right)=\pi \Gamma^{2} / \sigma$, as state I has a free

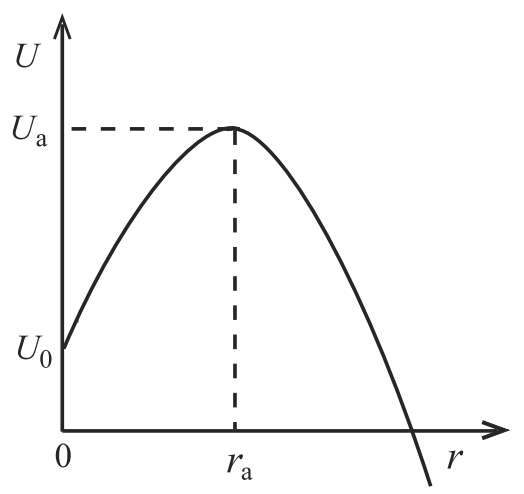

FIG. 4. Illustration of the free energy of a pre-pore as a function of the hydrophilic pre-pore radius, $r$. energy of $U(0)=U_{0}$. Therefore, the total activation energy for the transition from state $\mathrm{O}$ to pore state $\mathrm{P}$ can be described using Eq. (4).

Evans and colleagues investigated the effect of loading rate on the rupture tension of a GUV using dynamic tension spectroscopy. Based on their results, they predicted the existence of a metastable intermediate state, which supports model A. ${ }^{3}$ Briels and colleagues determined the free energy profile for a pore in a dipalmitoylphosphatidylcholine membrane using atomistic molecular dynamics simulations and reported that no energy barrier exists between intact state $\mathrm{O}$ and state $\mathrm{I}$, which supports model B. ${ }^{13,14}$ Recently, Evans and Smith reported that the energy profile for a lipid membrane containing a pre-pore depends on the types of lipids composing the membrane. ${ }^{4}$ The results of the present study clearly indicate that a free energy which does not depend on tension, $U_{0}$, contributes significantly to $U_{\mathrm{a}}$ based on the experimental results (not based on interpretation). However, we cannot determine which model is correct due to the limited experimental results.

\section{CONCLUSION}

In the present study, we determined the activation energy of constant tension-induced pore formation. We also assessed the dependence of $U_{\mathrm{a}}$ on $\sigma$ (i.e., $U_{a}=U_{0}+\pi \Gamma^{2} / \sigma$ ) and determined the values of $U_{0}$ and $\Gamma$. Our data clearly indicate that the classical theory of pore formation is not entirely correct and a free energy which does not depend on tension contributes significantly to $U_{\mathrm{a}}$. These data provide valuable information that will facilitate complete elucidation of the mechanism of tension-induced pore formation.

\section{ACKNOWLEDGMENTS}

This work was supported in part by a Grant-in-Aid for Scientific Research (B) (No. 15H04361) from the Japan Society for the Promotion of Science (JSPS) to M.Y.

${ }^{1}$ O. Sandre, L. Moreaux, and F. Brochard-Wyard, Proc. Natl. Acad. Sci. U. S. A. 96, 10591 (1999).

${ }^{2}$ E. Karatekin, O. Sandre, H. Guitouni, N. Borghi, P.-H. Puech, and F. Brochard-Wyard, Biophys. J. 84, 1734 (2003).

${ }^{3}$ E. Evans, V. Heinrich, F. Ludwig, and W. Rawicz, Biophys. J. 85, 2342 (2003).

${ }^{4}$ E. Evans and B. A. Smith, New J. Phys. 13, 095010 (2011).

${ }^{5}$ V. Levadny, T. Tsuboi, M. Belaya, and M. Yamazaki, Langmuir 29, 3848 (2013).

${ }^{6}$ M. A. S. Karal, V. Levadny, T. Tsuboi, M. Belaya, and M. Yamazaki, Phys. Rev. E 92, 012708 (2015).

${ }^{7}$ J. D. Litster, Phys. Lett. A 53, 193 (1975).

${ }^{8}$ C. Taupin, M. Dvolaitzky, and C. Sauterey, Biochemistry 14, 4771 (1975).

${ }^{9}$ B. V. Deryagin and Y. V. Gutop, Kolloidn. Zh. 24, 370 (1962).

${ }^{10}$ T. Tanaka, R. Sano, Y. Yamashita, and M. Yamazaki, Langmuir 20, 9526 (2004).

${ }^{11}$ W. Rawictz, K. C. Olbrich, T. J. McIntosh, D. Needham, and E. Evans, Biophys. J. 79, 328 (2000).

${ }^{12}$ R. W. Glaser, S. L. Leikin, L. V. Chernomordik, V. F. Pastushenko, and A. I. Sokirko, Biochem. Biophys. Acta 940, 275 (1988).

${ }^{13}$ T. V. Tolpekina, W. K. den Otter, and W. J. Briels, J. Chem. Phys. 121, 12060 (2004).

${ }^{14} \mathrm{~J}$. Wohlert, W. K. den Otter, O. Edholm, and W. J. Briels, J. Chem. Phys. 124, 154905 (2006).

${ }^{15}$ Z.-J. Wang and D. Frenkel, J. Chem. Phys. 123, 154701 (2005). 